Research Article

\title{
On the Sum of Degree-Based Topological Indices of Rhombus-Type Silicate and Oxide Structures
}

\author{
Rong Qi, ${ }^{1}$ Haidar Ali $\left(\mathbb{D},{ }^{2}\right.$ Usman Babar, ${ }^{3}$ Jia-Bao Liu $\mathbb{D}^{4},{ }^{4}$ and Parvez Ali ${ }^{5}$ \\ ${ }^{1}$ School of Computer and Data Engineering, Bengbu College of Technology and Business, Bengbu 233000, China \\ ${ }^{2}$ Department of Mathematics, Riphah International University, Faisalabad Campus, Faisalabad, Pakistan \\ ${ }^{3}$ Department of Mathematics, Government College University, Lahore, Pakistan \\ ${ }^{4}$ School of Mathematics and Physics, Anhui Jianzhu University, Hefei 230601, China \\ ${ }^{5}$ Department of Mechanical Engineering, College of Engineering, Qassim University, Unaizah, Saudi Arabia \\ Correspondence should be addressed to Haidar Ali; haidar3830@gmail.com
}

Received 17 July 2021; Accepted 8 December 2021; Published 29 December 2021

Academic Editor: Giuseppe Gaetano Luciano

Copyright ( 2021 Rong Qi et al. This is an open access article distributed under the Creative Commons Attribution License, which permits unrestricted use, distribution, and reproduction in any medium, provided the original work is properly cited.

\section{Introduction and Preliminary Results}

The representation of a graph is expressed by numbers, polynomials, and matrices. Graphs have their own characteristics that may be calculated by topological indices, and under graph automorphism, the topology of graphs remains unchanged. Degree-based topological indices are exceptionally important in different classes of indices and take on a vital role in graphic theory and in particular in science.

Silicate is a chemical compound and has many commercial uses. It is used for the manufacture of different glass and ceramics organic compounds in large scale due to its cheapness and availability everywhere in the world. Silicates can be obtained from the Earth's crust. In general, solid silicates are well-characterized and stable. Silicates like sodium orthosilicate and metasilicate, which have alkali cations and tiny or chain-like anions, are water soluble. When crystallised from a solution, they generate multiple solid hydrates. Water glass, which is made up of soluble sodium silicates and combinations, is a significant industrial and home chemical. For the construction of networks rhombus oxide and silicate, we refer the readers to 10 . Rhombus silicate network $\operatorname{RHSL}(t)$ and rhombus oxide network $\operatorname{RHOX}(t)$ are shown in Figures 1 and 2, respectively.

In this article, $\mathscr{G}$ is considered a network with a $V(\mathscr{G})$ vertex set and an edge set of $E(\mathscr{G})$ and $d_{r}$ is the degree of vertex $r \in V(\mathscr{G})$. Let $S_{\mathscr{G}}(r)$ denote the sum of the degrees of all vertices adjacent to a vertex $r$. Graovac et al. defined fifth M-Zagreb indices as polynomials for a molecular graph [1], and these are characterized as follows.

Let $\mathscr{G}$ be a graph. Then,

$$
\begin{aligned}
& M_{1} G_{5}(\mathscr{G})=\sum_{r s \in E(\mathscr{G})}\left(S_{G}(r)+S_{G}(s)\right), \\
& M_{2} G_{5}(\mathscr{G})=\sum_{r s \in E(\mathscr{G})}\left(S_{G}(r)+S_{G}(s)\right) .
\end{aligned}
$$

V. R. Kulli [2], motivated by the above indices, described some new topological indices, and he named them as the fifth M-Zagreb indices of first and second type and fifth hyper-M-Zagreb indices of first and second type of a graph $\mathscr{G}$. They are defined as

$$
\begin{aligned}
M_{1}^{a} G_{5}(\mathscr{G}) & =\sum_{r s \in E(\mathscr{G})}\left(S_{G}(r)+S_{G}(s)\right)^{a}, \\
M_{2}^{a} G_{5}(\mathscr{G}) & =\sum_{r s \in E(\mathscr{G})}\left(S_{G}(r)+S_{G}(s)\right)^{a}, \\
H M_{1} G_{5}(\mathscr{G}) & =\sum_{r s \in E(\mathscr{G})}\left(S_{G}(r)+S_{G}(s)\right)^{2},
\end{aligned}
$$




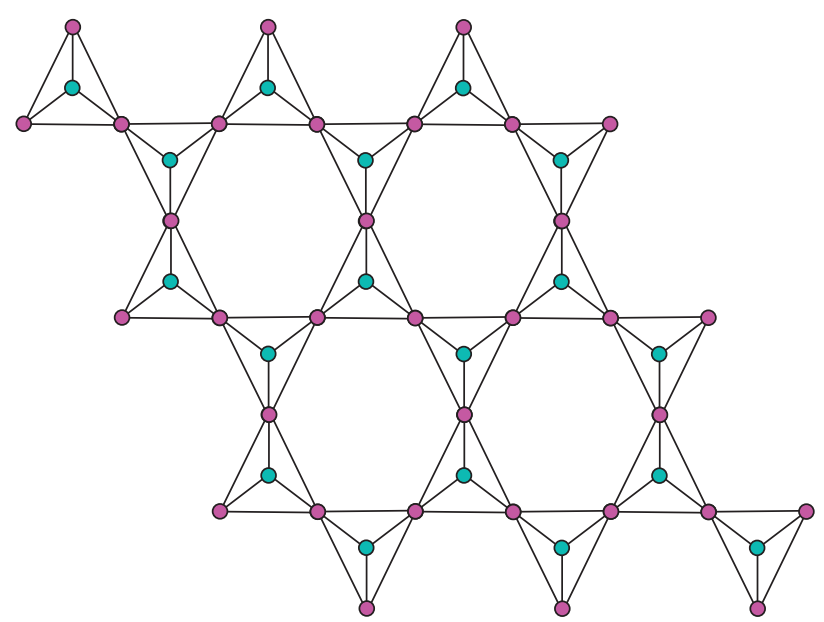

FIGURE 1: Graph of rhombus silicate network (RHSL $(t)$ ).

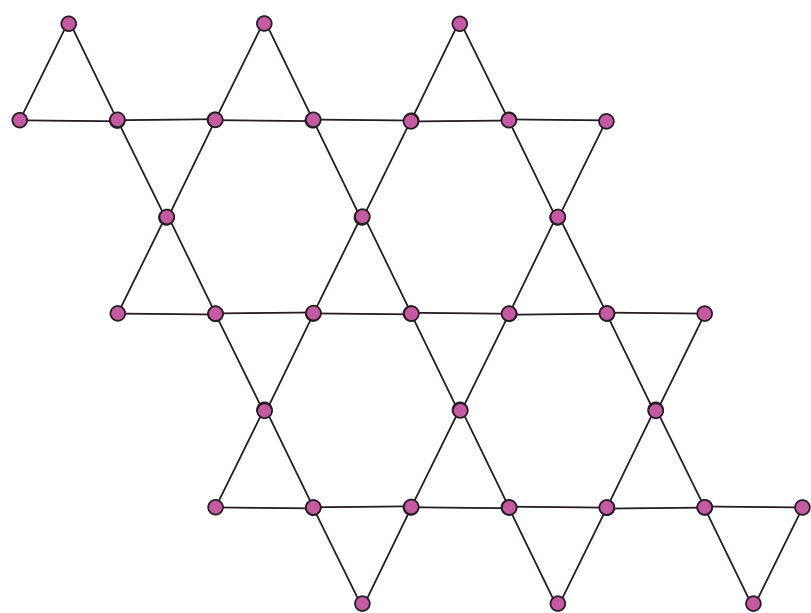

FIgURE 2: Graph of rhombus oxide network $(\operatorname{RHOX}(t))$.

$$
H M_{2} G_{5}(\mathscr{G})=\sum_{r s \in E(\mathscr{G})}\left(S_{G}(r)+S_{G}(s)\right)^{2} .
$$

They also define a new version of Zagreb index which is called as the third Zagreb index or fifth $M_{3}$-Zagreb [3].

$$
M_{3} G_{5}(\mathscr{G})=\sum_{r s \in E(\mathscr{G})}\left|S_{G}(r)-S_{G}(s)\right| .
$$

Corresponding to the above indices, he defined the general fifth $M_{1}$-Zagreb polynomial and the general fifth $M_{2}$-Zagreb polynomial of a molecular graph $\mathscr{G}$ as

$$
\begin{aligned}
& M_{1}^{a} G_{5}(\mathscr{G}, x)=\prod_{r s \in E(\mathscr{G})} x^{\left(S_{G}(r)+S_{G}(s)\right)^{a}}, \\
& M_{2}^{a} G_{5}(\mathscr{G}, x)=\prod_{r s \in E(\mathscr{G})} x^{\left(S_{G}(r)+S_{G}(s)\right)^{a}} .
\end{aligned}
$$

The fifth $M_{1}$ - and $M_{2}$ - Zagreb polynomials of a graph are defined as

$$
\begin{aligned}
& M_{1} G_{5}(\mathscr{G}, x)=\prod_{r s \in E(\mathscr{G})} x^{\left(S_{G}(r)+S_{G}(s)\right)}, \\
& M_{2} G_{5}(\mathscr{G}, x)=\prod_{r s \in E(\mathscr{G})} x^{\left(S_{G}(r)+S_{G}(s)\right)} .
\end{aligned}
$$

The fifth $H M_{1}$ and $H M_{2}$ Zagreb polynomials of the graph are defined as

$$
\begin{aligned}
& H M_{1} G_{5}(\mathscr{G}, x)=\prod_{r s \in E(\mathscr{G})} x^{\left(S_{G}(r)+S_{G}(s)\right)^{2}}, \\
& H M_{2} G_{5}(\mathscr{G}, x)=\prod_{r s \in E(\mathscr{G})} x^{\left(S_{G}(r)+S_{G}(s)\right)^{2}} .
\end{aligned}
$$




\section{Main Results}

We have studied the topological indices introduced by Kulli $[2,4]$ named as fifth $\mathrm{M}$-Zagreb indices, fifth $\mathrm{M}$-Zagreb polynomials, and $M_{3}-Z a g r e b$ index and computed exact formulae of these indices for rhombus-type silicate and oxide networks. Ali et al. studied degree-based topological indices for various networks [5-8]. For the basic notations and definitions, see [9-11].

2.1. Results for the Rhombus Type of Silicate Networks. In this section, we calculate degree-based topological indices of the dimension $t$ for rhombus-type silicate networks. In the following theorems, we compute $M$-Zagreb indices and polynomials.

Theorem 2.1.1. Let $\mathscr{G}_{1} \cong R H S L(t)$ be the rhombus-type silicate network; then, the first and second fifth M-Zagreb indices are equal to

$$
\begin{aligned}
& M_{1} G_{5}\left(\mathscr{G}_{1}\right)=36\left(1-10 t+18 t^{2}\right), \\
& M_{2} G_{5}\left(\mathscr{G}_{1}\right)=18\left(119-490 t+480 t^{2}\right) .
\end{aligned}
$$

Proof. The outcome can be obtained by using the edge partition in Table 1.

By using equation [5],

$$
\begin{aligned}
M_{1} G_{5}\left(\mathscr{G}_{1}\right)= & \sum_{r s \in E\left(\mathscr{G}_{1}\right)}\left(S_{G}(r)+S_{G}(s)\right), \\
M_{1} G_{5}\left(\mathscr{G}_{1}\right)= & (12+12)\left|E_{1}\left(\mathscr{G}_{1}(t)\right)\right|+(12+24)\left|E_{2}\left(\mathscr{G}_{1}(t)\right)\right|+(15+15)\left|E_{3}\left(\mathscr{G}_{1}(t)\right)\right|+(15+24)\left|E_{4}\left(\mathscr{G}_{1}(t)\right)\right| \\
& +(15+27)\left|E_{5}\left(\mathscr{G}_{1}(t)\right)\right|+(18+24)\left|E_{6}\left(\mathscr{G}_{1}(t)\right)\right|+(18+27)\left|E_{7}\left(\mathscr{G}_{1}(t)\right)\right| \\
& +(18+30)\left|E_{8}\left(\mathscr{G}_{1}(t)\right)\right|+(24+27)\left|E_{9}\left(\mathscr{G}_{1}(t)\right)\right|+(27+27)\left|E_{10}\left(\mathscr{G}_{1}(t)\right)\right|+(27+30)\left|E_{11}\left(\mathscr{G}_{1}(t)\right)\right| \\
& +(30+30)\left|E_{12}\left(\mathscr{G}_{1}(t)\right)\right|, \\
= & (12+12)(6)+(12+24)(6)+(15+15)(4 t-4)+(15+24)(8)+(15+27)(16 t-24) \\
& +(18+24)(2)+(18+27)(8 t-12)+(18+30)\left(6 t^{2}-20 t+16\right)+(24+27)(8) \\
& +(27+27)(8 t-14)+(27+30)(8 t-16)+(30+30)\left(6 t^{2}-24 t+24\right) .
\end{aligned}
$$

By doing some calculations, we obtain

$$
M_{1} G_{5}\left(\mathscr{G}_{1}\right)=36\left(1-10 t+18 t^{2}\right) .
$$

$$
\begin{aligned}
M_{2} G_{5}(\mathscr{G})= & \sum_{r s \in E\left(\mathscr{G}_{1}\right)}\left(S_{G}(r)+S_{G}(s)\right), \\
M_{2} G_{5}\left(\mathscr{G}_{1}\right)= & (12 \times 12)\left|E_{1}\left(\mathscr{G}_{1}(t)\right)\right|+(12 \times 24)\left|E_{2}\left(\mathscr{G}_{1}(t)\right)\right|+(15 \times 15)\left|E_{3}\left(\mathscr{G}_{1}(t)\right)\right|+(15 \times 24)\left|E_{4}\left(\mathscr{G}_{1}(t)\right)\right| \\
& +(15 \times 27)\left|E_{5}\left(\mathscr{G}_{1}(t)\right)\right|+(18 \times 24)\left|E_{6}\left(\mathscr{G}_{1}(t)\right)\right|+(18 \times 27)\left|E_{7}\left(\mathscr{G}_{1}(t)\right)\right| \\
& +(18 \times 30)\left|E_{8}\left(\mathscr{G}_{1}(t)\right)\right|+(24 \times 27)\left|E_{9}\left(\mathscr{G}_{1}(t)\right)\right|+(27 \times 27)\left|E_{10}\left(\mathscr{G}_{1}(t)\right)\right|+(27 \times 30)\left|E_{11}\left(\mathscr{G}_{1}(t)\right)\right| \\
& +(30 \times 30)\left|E_{12}\left(\mathscr{G}_{1}(t)\right)\right|, \\
= & (12 \times 12)(6)+(12 \times 24)(6)+(15 \times 15)(4 t-4)+(15 \times 24)(8)+(15 \times 27)(16 t-24) \\
& +(18 \times 24)(2)+(18 \times 27)(8 t-12)+(18 \times 30)\left(6 t^{2}-20 t+16\right)+(24 \times 27)(8) \\
& +(27 \times 27)(8 t-14)+(27 \times 30)(8 t-16)+(30 \times 30)\left(6 t^{2}-24 t+24\right) .
\end{aligned}
$$

By doing some calculations, we obtain

$$
M_{2} G_{5}\left(\mathscr{G}_{1}\right)=18\left(119-490 t+480 t^{2}\right) \text {. }
$$

Theorem 2.1.2. Consider the rhombus-type silicate network $\mathscr{G}_{1} \cong R H S L(t)$ for $t \in \mathbb{N}$. Then, the first and second general fifth $M$-Zagreb indices are equal to 
TABLE 1: Edge partition of rhombus-type silicate network $(R H S L(t))$ based on sum of degrees of end vertices of each edge.

$\left(S_{r}, S_{s}\right)$

Where rs $\in E\left(\mathscr{G}_{1}\right)$

$(12,12)$

$(12,24)$

$(15,15)$

$(15,24)$

$(15,27)$

$(18,24)$

Where $r s \in E\left(\mathscr{G}_{1}\right)$

$(18,27)$

$(18,30)$

$(24,27)$

$(27,27)$

$(27,30)$

$(30,30)$
Number of edges

$$
\begin{gathered}
6 \\
6 \\
4 t-4 \\
8 \\
16 t-24 \\
2 \\
\\
8 t-2 \\
6 t^{2}-20 t+16 \\
8 \\
8 t-14 \\
8 t-16 \\
6 t^{2}-24 t+24
\end{gathered}
$$

Proof. Let $\mathscr{G}_{1}$ be the rhombus-type silicate network. Table 1 shows such an edge partition of $R H S L(t)$. Thus, from [9], it follows that

$$
M_{1}^{a} G_{5}(\mathscr{G})=\sum_{r s \in E(\mathscr{G})}\left(S_{G}(r)+S_{G}(s)\right)^{a}
$$

By using edge partitions in Table 1, we obtain

$$
\begin{aligned}
M_{1}^{a} G_{5}\left(\mathscr{G}_{1}\right)= & (12+12)^{a}\left|E_{1}\left(\mathscr{G}_{1}(t)\right)\right|+(12+24)^{a}\left|E_{2}\left(\mathscr{G}_{1}(t)\right)\right|+(15+15)^{a}\left|E_{3}\left(\mathscr{G}_{1}(t)\right)\right| \\
& +(15+24)^{a}\left|E_{4}\left(\mathscr{G}_{1}(t)\right)\right|+(15+27)^{a}\left|E_{5}\left(\mathscr{G}_{1}(t)\right)\right|+(18+24)^{a}\left|E_{6}\left(\mathscr{G}_{1}(t)\right)\right|+(18+27)^{a}\left|E_{7}\left(\mathscr{G}_{1}(t)\right)\right| \\
& +(18+30)^{a}\left|E_{8}\left(\mathscr{G}_{1}(t)\right)\right|+(24+27)^{a}\left|E_{9}\left(\mathscr{G}_{1}(t)\right)\right|+(27+27)^{a}\left|E_{10}\left(\mathscr{G}_{1}(t)\right)\right| \\
& +(27+30)^{a}\left|E_{11}\left(\mathscr{G}_{1}(t)\right)\right|+(30+30)^{a}\left|E_{12}\left(\mathscr{G}_{1}(t)\right)\right| \\
= & (12+12)^{a}(6)+(12+24)^{a}(6)+(15+15)^{a}(4 t-4)+(15+24)^{a}(8)+(15+24)^{a}(16 t-24) \\
& +(18+24)^{a}(2)+(18+27)^{a}(8 t-12)+(18+30)^{a}\left(6 t^{2}-20 t+16\right)+(24+27)^{a}(8) \\
& +(27+27)^{a}(8 t-14)+(27+30)^{a}(8 t-16)+(30+30)^{a}\left(6 t^{2}-24 t+24\right) .
\end{aligned}
$$

By doing some calculations, we have

$$
\begin{aligned}
M_{1}^{a} G_{5}\left(\mathscr{G}_{1}\right)= & {\left[\left(2^{1+3 a} 3^{1+a}+2^{3+2 a} 3^{1+a} 5^{a}-4 \times 3^{1+2 a} 5^{a}+6^{1+2 a}-2^{3+a} 3^{1+a} 7^{a}-2^{2+a} 15^{a}+3^{a} 16^{1+a}+2^{1+a} 21^{a}\right.\right.} \\
& -7 \times 2^{1+a} 27^{a}+8 \times 39^{a}+8 \times 51^{a}-16 \times 57^{a}+t\left(-5 \times 3^{a} 4^{1+2 a}-2^{3+2 a} 3^{1+a} 5^{a}+2^{2+a} 15^{a}\right. \\
& \left.\left.\left.+2^{4+a} 21^{a}+2^{3+a} 27^{a}+8 \times 45^{a}+8 \times 57^{a}\right)+t^{2}\left(2^{1+4 a} 3^{1+a}+2^{1+2 a} 3^{1+a} 5^{a}\right)\right)\right] .
\end{aligned}
$$

From [12], we have 


$$
M_{2}^{a} G_{5}(\mathscr{G})=\sum_{r s \in E(\mathscr{G})}\left(S_{G}(r)+S_{G}(s)\right)^{a}
$$

By using edge partitions in Table 1, we obtain

$$
\begin{aligned}
M_{2}^{a} G_{5}\left(\mathscr{G}_{1}\right)= & (12 \times 12)^{a}\left|E_{1}\left(\mathscr{G}_{1}(t)\right)\right|+(12 \times 24)^{a}\left|E_{2}\left(\mathscr{G}_{1}(t)\right)\right|+(15 \times 15)^{a}\left|E_{3}\left(\mathscr{G}_{1}(t)\right)\right|+(15 \times 24)^{a} \mid \\
& E_{4}\left(\mathscr{G}_{1}(t)\right)\left|+(15 \times 27)^{a}\right| E_{5}\left(\mathscr{G}_{1}(t)\right)\left|+(18 \times 24)^{a}\right| E_{6}\left(\mathscr{G}_{1}(t)\right)\left|+(18 \times 27)^{a}\right| E_{7}\left(\mathscr{G}_{1}(t)\right) \mid \\
& +(18 \times 30)^{a}\left|E_{8}\left(\mathscr{G}_{1}(t)\right)\right|+(24 \times 27)^{a}\left|E_{9}\left(\mathscr{G}_{1}(t)\right)\right|+(27 \times 27)^{a}\left|E_{10}\left(\mathscr{G}_{1}(t)\right)\right|+(27 \times 30)^{a} \\
& \left|E_{11}\left(\mathscr{G}_{1}(t)\right)\right|+(30 \times 30)^{a}\left|E_{12}\left(\mathscr{G}_{1}(t)\right)\right|, \\
= & (12 \times 12)^{a}(6)+(12 \times 24)^{a}(6)+(15 \times 15)^{a}(4 t-4)+(15 \times 24)^{a}(8)+(15 \times 24)^{a}(16 t-24) \\
& +(18 \times 24)^{a}(2)+(18 \times 27)^{a}(8 t-12)+(18 \times 30)^{a}\left(6 t^{2}-20 t+16\right)+(24 \times 27)^{a} \\
& (8)+(27 \times 27)^{a}(8 t-14)+(27 \times 30)^{a}(8 t-16)+(30 \times 30)^{a}\left(6 t^{2}-24 t+24\right) .
\end{aligned}
$$

By doing some calculations, we have

$$
\begin{aligned}
M_{2}^{a} G_{5}\left(\mathscr{G}_{1}\right)= & {\left[\left(2^{1+4 a} 3^{1+2 a}+2^{1+5 a} 3^{1+2 a}-2^{2+a} 3^{1+5 a}-8 \times 3^{1+4 a} 5^{a}+2^{3+2 a} 3^{1+2 a} 25^{a}+2^{1+4 a} 27^{a}+8^{1+a} 45^{a}+8^{1+a} 81^{a}\right.\right.} \\
& +4^{2+a} 135^{a}-4 \times 225^{a}-2^{4+a} 405^{a}-14 \times 729^{a}+t\left(-2^{3+2 a} 3^{1+2 a} 25^{a}-20^{1+a} 27^{a}+4 \times 225^{a}+2^{3+a} 243^{a}\right. \\
& \left.\left.\left.+16 \times 405^{a}+2^{3+a} 405^{a}+8 \times 729^{a}\right)+t^{2}\left(2^{1+2 a} 3^{1+3 a} 5^{a}+6^{1+2 a} 25^{a}\right)\right)\right] .
\end{aligned}
$$

Theorem 2.1.3. Consider the rhombus-type silicate network $\mathscr{G}_{1} \cong R H S L(t)$ for $t \in \mathbb{N}$. Then, the first and second hyperfifth $M$-Zagreb indices are equal to

$$
\begin{aligned}
& H M_{1} G_{5}\left(\mathscr{G}_{1}\right)=36\left(221-976 t+984 t^{2}\right), \\
& H M_{2} G_{5}\left(\mathscr{G}_{1}\right)=162\left(28307-68242 t+40800 t^{2}\right) .
\end{aligned}
$$

Proof. Let $\mathscr{G}_{1}$ be the rhombus type of silicate network. Table 1 shows such an edge partition of $R H S L(t)$. Thus, from [13], it follows that

$$
H M_{1} G_{5}(\mathscr{G})=\sum_{r s \in E(\mathscr{G})}\left(S_{G}(r)+S_{G}(s)\right)^{2} .
$$

By using edge partitions in Table 1, we obtain

$$
\begin{aligned}
H M_{1} G_{5}\left(\mathscr{G}_{1}\right)= & (12 \times 12)^{2}\left|E_{1}\left(\mathscr{G}_{1}(t)\right)\right|+(12 \times 24)^{2}\left|E_{2}\left(\mathscr{G}_{1}(t)\right)\right|+(15 \times 15)^{2}\left|E_{3}\left(\mathscr{G}_{1}(t)\right)\right|+(15 \times 24)^{2} \mid \\
& E_{4}\left(\mathscr{G}_{1}(t)\right)\left|+(15 \times 27)^{2}\right| E_{5}\left(\mathscr{G}_{1}(t)\right)\left|+(18 \times 24)^{2}\right| E_{6}\left(\mathscr{G}_{1}(t)\right)\left|+(18 \times 27)^{2}\right| E_{7}\left(\mathscr{G}_{1}(t)\right) \mid \\
& +(18 \times 30)^{2}\left|E_{8}\left(\mathscr{G}_{1}(t)\right)\right|+(24 \times 27)^{2}\left|E_{9}\left(\mathscr{G}_{1}(t)\right)\right|+(27 \times 27)^{2}\left|E_{10}\left(\mathscr{G}_{1}(t)\right)\right|+(27 \times 30)^{2} \\
& \left|E_{11}\left(\mathscr{G}_{1}(t)\right)\right|+(30 \times 30)^{2}\left|E_{12}\left(\mathscr{G}_{1}(t)\right)\right|, \\
= & (12 \times 12)^{2}(6)+(12 \times 24)^{2}(6)+(15 \times 15)^{2}(4 t-4)+(15 \times 24)^{2}(8)+(15 \times 24)^{2}(16 t-24) \\
& +(18 \times 24)^{2}(2)+(18 \times 27)^{2}(8 t-12)+(18 \times 30)^{2}\left(6 t^{2}-20 t+16\right)+(24 \times 27)^{2} \\
& (8)+(27 \times 27)^{2}(8 t-14)+(27 \times 30)^{2}(8 t-16)+(30 \times 30)^{2}\left(6 t^{2}-24 t+24\right) .
\end{aligned}
$$

By doing some calculations, we have

$$
H M_{1} G_{5}\left(\mathscr{G}_{1}\right)=36\left(221-976 t+984 t^{2}\right) \text {. }
$$

From [14], we have

$$
H M_{2}^{2} G_{5}(\mathscr{G})=\sum_{r s \in E(\mathscr{G})}\left(S_{G}(r)+S_{G}(s)\right)^{2} .
$$

By using edge partitions in Table 1, we obtain 


$$
\begin{aligned}
H M_{2} G_{5}\left(\mathscr{G}_{1}\right)= & (12 \times 12)^{2}\left|E_{1}\left(\mathscr{G}_{1}(t)\right)\right|+(12 \times 24)^{2}\left|E_{2}\left(\mathscr{G}_{1}(t)\right)\right|+(15 \times 15)^{2}\left|E_{3}\left(\mathscr{G}_{1}(t)\right)\right| \\
& +(15 \times 24)^{2}\left|E_{4}\left(\mathscr{G}_{1}(t)\right)\right|+(15 \times 27)^{2}\left|E_{5}\left(\mathscr{G}_{1}(t)\right)\right|+(18 \times 24)^{2}\left|E_{6}\left(\mathscr{G}_{1}(t)\right)\right| \\
& +(18 \times 27)^{2}\left|E_{7}\left(\mathscr{G}_{1}(t)\right)\right|+(18 \times 30)^{2}\left|E_{8}\left(\mathscr{G}_{1}(t)\right)\right|+(24 \times 27)^{2}\left|E_{9}\left(\mathscr{G}_{1}(t)\right)\right| \\
& +(27 \times 27)^{2}\left|E_{10}\left(\mathscr{G}_{1}(t)\right)\right|+(27 \times 30)^{2}\left|E_{11}\left(\mathscr{G}_{1}(t)\right)\right|+(30 \times 30)^{2}\left|E_{12}\left(\mathscr{G}_{1}(t)\right)\right|, \\
= & (12 \times 12)^{2}(6)+(12 \times 24)^{2}(6)+(15 \times 15)^{2}(4 t-4)+(15 \times 24)^{2}(8) \\
& +(15 \times 24)^{2}(16 t-24)+(18 \times 24)^{2}(2)+(18 \times 27)^{2}(8 t-12)+(18 \times 30)^{2}\left(6 t^{2}-20 t+16\right) \\
& +(24 \times 27)^{2}(8)+(27 \times 27)^{2}(8 t-14)+(27 \times 30)^{2}(8 t-16)+(30 \times 30)^{2}\left(6 t^{2}-24 t+24\right) .
\end{aligned}
$$

By doing some calculations, we have

$$
H M_{2}^{a} G_{5}\left(\mathscr{G}_{1}\right)=162\left(28307-68242 t+40800 t^{2}\right) \text {. }
$$

Theorem 2.1.4. Consider the rhombus-type silicate network $\mathscr{G}_{1} \cong R H S L(t)$ for $t \in \mathbb{N}$. Then, the third M-Zagreb index is equal to

$$
M_{3} G_{5}\left(\mathscr{G}_{1}\right)=\left(-232+248 t+12 t^{2}\right) \text {. }
$$

Proof. Let $\mathscr{G}_{1}$ be the rhombus silicate network. Table 1 shows such an edge partition of RHSL $(t)$. Thus, from [15], it follows that

$$
M_{3} G_{5}(\mathscr{G})=\sum_{r s \in E(\mathscr{G})}\left|S_{G}(r)+S_{G}(s)\right| .
$$

By using edge partitions in Table 1, we obtain

$$
\begin{aligned}
M_{3} G_{5}\left(\mathscr{G}_{1}\right)= & \left|12-12 \| E_{1}\left(\mathscr{G}_{1}(t)\right)\right|+|12-24|\left|E_{2}\left(\mathscr{G}_{1}(t)\right)\right|+|15-15|\left|E_{3}\left(\mathscr{G}_{1}(t)\right)\right|+|15-24|\left|E_{4}\left(\mathscr{G}_{1}(t)\right)\right| \\
& +|15-27|\left|E_{5}\left(\mathscr{G}_{1}(t)\right)\right|+|18-24|\left|E_{6}\left(\mathscr{G}_{1}(t)\right)\right|+|18-27|\left|E_{7}\left(\mathscr{G}_{1}(t)\right)\right|+|18-30|\left|E_{8}\left(\mathscr{G}_{1}(t)\right)\right| \\
& +|24-27|\left|E_{9}\left(\mathscr{G}_{1}(t)\right)\right|+|27-27|\left|E_{10}\left(\mathscr{G}_{1}(t)\right)\right|+\left|27-30\left\|\left|E_{11}\left(\mathscr{G}_{1}(t)\right)\right|+\left|30-30 \| E_{12}\left(\mathscr{G}_{1}(t)\right)\right|,\right.\right. \\
= & |12-12|(6)+|12-24|(6)+|15-15|(4 t-4)+|15-24|(8)+|15-27|(16 t-24)+\mid 18 \\
& -24|(2)+| 18-27|(8 t-12)+| 18-30\left|\left(6 t^{2}-20 t+16\right)+\right| 24-27|(8)+| 27-27 \mid(8 t-14) \\
& +|27-30|(8 t-16)+|30-30|\left(6 t^{2}-24 t+24\right) .
\end{aligned}
$$

By doing some calculations, we have

$$
M_{3} G_{5}\left(\mathscr{G}_{1}\right)=\left(-232+248 t+12 t^{2}\right) \text {. }
$$

Theorem 2.1.5. Let $\mathscr{G}_{1} \cong R H S L(t)$ be the first type of rhombus-type silicate network; then, general fifth $M$-Zagreb polynomials of first and second type are equal to

Corresponding to the above indices, we are going to compute general fifth $M$-Zagreb polynomials for rhombustype silicate network RHSL $(t)$.

$$
\begin{aligned}
M_{1}^{a} G_{5}\left(\mathscr{G}_{1}, x\right)= & 6 x^{24^{a}}+(4 t-4) x^{30^{a}}+6 x^{36^{a}}+8 x^{39^{a}}+(16 t-22) x^{42^{a}}+(8 t-12) x^{45^{a}} \\
& +\left(6 t^{2}-20 t+16\right) x^{48^{a}}+8 x^{51^{a}}+(8 t-14) x^{54^{a}}+(8 t-16) x^{57^{a}}+\left(6 t^{2}-24 t+24\right) x^{60^{a}}, \\
M_{2}^{a} G_{5}\left(\mathscr{G}_{1}, x\right)= & 6 x^{144^{a}}+(4 t-4) x^{225^{a}}+6 x^{288^{a}}+8 x^{360^{a}}+8(2 t-3) x^{405^{a}}+2 x^{432^{a}}+4(2 t-3) x^{486^{a}} \\
& +2(t-2)(3 t-4) x^{540^{a}}+8 x^{648^{a}}+2(4 t-7) x^{729^{a}}+8(t-2) x^{810^{a}}+6(t-2)^{2} x^{900^{a}} .
\end{aligned}
$$

Proof. We obtain the outcome with the edge partition in

Table 1. It follows from [1] that 


$$
\begin{aligned}
M_{1}^{a} G_{5}\left(\mathscr{G}_{1}, x\right)= & \sum_{r s \in E\left(\mathscr{G}_{1}\right)} x^{\left(S_{G}(r)+S_{G}(s)\right)^{a}}, \\
M_{1}^{a} G_{5}\left(\mathscr{G}_{1}, x\right)= & x^{(12+12)^{a}}\left|E_{1}\left(\mathscr{G}_{1}(t)\right)\right|+x^{(12+24)^{a}}\left|E_{2}\left(\mathscr{G}_{1}(t)\right)\right|+x^{(15+15)^{a}}\left|E_{3}\left(\mathscr{G}_{1}(t)\right)\right|+x^{(15+24)^{a}}\left|E_{4}\left(\mathscr{G}_{1}(t)\right)\right| \\
& +x^{(15+27)^{a}}\left|E_{5}\left(\mathscr{G}_{1}(t)\right)\right|+x^{(18+24)^{a}}\left|E_{6}\left(\mathscr{G}_{1}(t)\right)\right|+x^{(18+27)^{a}}\left|E_{7}\left(\mathscr{G}_{1}(t)\right)\right|+x^{(18+30)^{a}}\left|E_{8}\left(\mathscr{G}_{1}(t)\right)\right| \\
& +x^{(24+27)^{a}}\left|E_{9}\left(\mathscr{G}_{1}(t)\right)\right|+x^{(27+27)^{a}}\left|E_{10}\left(\mathscr{G}_{1}(t)\right)\right|+x^{(27+30)^{a}}\left|E_{11}\left(\mathscr{G}_{1}(t)\right)\right|+x^{(30+30)^{a}}\left|E_{12}\left(\mathscr{G}_{1}(t)\right)\right|, \\
& M_{1}^{a} G_{5}\left(\mathscr{G}_{1}, x\right)=x^{(12+12)^{a}}(6)+x^{(12+24)^{a}}(6)+x^{(15+15)^{a}}(4 t-4)+x^{(15+24)^{a}}(8) \\
& +x^{(15+27)^{a}}(16 t-24)+x^{(18+24)^{a}}(2)+x^{(18+27)^{a}}(8 t-12)+x^{(18+30)^{a}}\left(6 t^{2}-20 t+16\right) \\
& +x^{(24+27)^{a}}(8)+x^{(27+27)^{a}}(8 t-14)+x^{(27+30)^{a}}(8 t-16)+x^{(30+30)^{a}}\left(6 t^{2}-24 t+24\right) .
\end{aligned}
$$

By doing some calculations, we obtain

$$
\begin{aligned}
M_{1}^{a} G_{5}\left(\mathscr{G}_{1}, x\right)= & 6 x^{24^{a}}+(4 t-4) x^{30^{a}}+6 x^{36^{a}}+8 x^{39^{a}}+(16 t-22) x^{42^{a}}+(8 t-12) x^{45^{a}} \\
& +\left(6 t^{2}-20 t+16\right) x^{48^{a}}+8 x^{51^{a}}+(8 t-14) x^{54^{a}}+(8 t-16) x^{57^{a}}+\left(6 t^{2}-24 t+24\right) x^{60^{a}} .
\end{aligned}
$$

Also, from [3],

$$
\begin{aligned}
M_{2}^{a} G_{5}\left(\mathscr{G}_{1}, x\right)= & \sum_{r s \in E\left(\mathscr{G}_{1}\right)} x^{\left(S_{G}(r)+S_{G}(s)\right)^{a}}, \\
M_{2}^{a} G_{5}\left(\mathscr{G}_{1}, x\right)= & x^{(12+12)^{a}}\left|E_{1}\left(\mathscr{G}_{1}(t)\right)\right|+x^{(12+24)^{a}}\left|E_{2}\left(\mathscr{G}_{1}(t)\right)\right|+x^{(15+15)^{a}}\left|E_{3}\left(\mathscr{G}_{1}(t)\right)\right|+x^{(15+24)^{a}}\left|E_{4}\left(\mathscr{G}_{1}(t)\right)\right| \\
& +x^{(15+27)^{a}}\left|E_{5}\left(\mathscr{G}_{1}(t)\right)\right|+x^{(18+24)^{a}}\left|E_{6}\left(\mathscr{G}_{1}(t)\right)\right|+x^{(18+27)^{a}}\left|E_{7}\left(\mathscr{G}_{1}(t)\right)\right|+x^{(18+30)^{a}}\left|E_{8}\left(\mathscr{G}_{1}(t)\right)\right| \\
& +x^{(24+27)^{a}}\left|E_{9}\left(\mathscr{G}_{1}(t)\right)\right|+x^{(27+27)^{a}}\left|E_{10}\left(\mathscr{G}_{1}(t)\right)\right|+x^{(27+30)^{a}}\left|E_{11}\left(\mathscr{G}_{1}(t)\right)\right|+x^{(30+30)^{a}}\left|E_{12}\left(\mathscr{G}_{1}(t)\right)\right|, \\
& M_{2}^{a} G_{5}\left(\mathscr{G}_{1}, x\right)=x^{(12+12)^{a}}(6)+x^{(12+24)^{a}}(6)+x^{(15+15)^{a}}(4 t-4)+x^{(15+24)^{a}}(8) \\
& +x^{(15+27)^{a}}(16 t-24)+x^{(18+24)^{a}}(2)+x^{(18+27)^{a}}(8 t-12)+x^{(18+30)^{a}}\left(6 t^{2}-20 t+16\right) \\
& +x^{(24+27)^{a}}(8)+x^{(27+27)^{a}}(8 t-14)+x^{(27+30)^{a}}(8 t-16)+x^{(30+30)^{a}}\left(6 t^{2}-24 t+24\right) .
\end{aligned}
$$

By making some calculations, we obtain

$$
\begin{aligned}
M_{2}^{a} G_{5}\left(\mathscr{G}_{1}, x\right)= & 6 x^{144^{a}}+(4 t-4) x^{225^{a}}+6 x^{288^{a}}+8 x^{360^{a}}+8(2 t-3) x^{405^{a}}+2 x^{432^{a}}+4(2 t-3) \\
& x^{486^{a}}+2(t-2)(3 t-4) x^{540^{a}}+8 x^{648^{a}}+2(4 t-7) x^{729^{a}}+8(t-2) x^{810^{a}}+6(t-2)^{2} x^{900^{a}} .
\end{aligned}
$$

Corresponding to the above indices, we are going to compute fifth $M$-Zagreb polynomials for rhombus-type silicate network RHSL $(t)$.
Theorem 2.1.6. Let $\mathscr{G}_{1} \cong R H S L(t)$ be the rhombus type of silicate network; then, fifth $M$-Zagreb polynomials of first and second type are equal to 


$$
\begin{aligned}
M_{1} G_{5}\left(\mathscr{G}_{1}, x\right)= & 6 x^{24}+(4 t-4) x^{30}+6 x^{36}+8 x^{39}+(16 t-22) x^{42}+(8 t-12) x^{45} \\
& +\left(6 t^{2}-20 t+16\right) x^{48}+8 x^{51}+(8 t-14) x^{54}+(8 t-16) x^{57}+\left(6 t^{2}-24 t+24\right) x^{60} \\
M_{2} G_{5}\left(\mathscr{G}_{1}, x\right)= & 6 x^{144}+(4 t-4) x^{225}+6 x^{288}+8 x^{360}+8(2 t-3) x^{405}+2 x^{432}+4(2 t-3) x^{486} \\
& +2(t-2)(3 t-4) x^{540}+8 x^{648}+2(4 t-7) x^{729}+8(t-2) x^{810}+6(t-2)^{2} x^{900} .
\end{aligned}
$$

Proof. We obtain the outcome with the edge partition in

Table 1. It follows from [16] that

$$
\begin{aligned}
M_{1} G_{5}\left(\mathscr{G}_{1}, x\right)= & \sum_{r s \in E\left(\mathscr{G}_{1}\right)} x^{\left(S_{G}(r)+S_{G}(s)\right)}, \\
M_{1} G_{5}\left(\mathscr{G}_{1}, x\right)= & x^{(12+12)}\left|E_{1}\left(\mathscr{G}_{1}(t)\right)\right|+x^{(12+24)}\left|E_{2}\left(\mathscr{G}_{1}(t)\right)\right|+x^{(15+15)}\left|E_{3}\left(\mathscr{G}_{1}(t)\right)\right|+x^{(15+24)}\left|E_{4}\left(\mathscr{G}_{1}(t)\right)\right| \\
& +x^{(15+27)}\left|E_{5}\left(\mathscr{G}_{1}(t)\right)\right|+x^{(18+24)}\left|E_{6}\left(\mathscr{G}_{1}(t)\right)\right|+x^{(18+27)}\left|E_{7}\left(\mathscr{G}_{1}(t)\right)\right|+x^{(18+30)}\left|E_{8}\left(\mathscr{G}_{1}(t)\right)\right| \\
& +x^{(24+27)}\left|E_{9}\left(\mathscr{G}_{1}(t)\right)\right|+x^{(27+27)}\left|E_{10}\left(\mathscr{G}_{1}(t)\right)\right|+x^{(27+30)}\left|E_{11}\left(\mathscr{G}_{1}(t)\right)\right|+x^{(30+30)}\left|E_{12}\left(\mathscr{G}_{1}(t)\right)\right|, \\
= & x^{(12+12)}(6)+x^{(12+24)}(6)+x^{(15+15)}(4 t-4)+x^{(15+24)}(8) \\
& +x^{(15+27)}(16 t-24)+x^{(18+24)}(2)+x^{(18+27)}(8 t-12)+x^{(18+30)}\left(6 t^{2}-20 t+16\right) \\
& +x^{(24+27)}(8)+x^{(27+27)}(8 t-14)+x^{(27+30)}(8 t-16)+x^{(30+30)}\left(6 t^{2}-24 t+24\right) .
\end{aligned}
$$

By doing some calculations, we obtain

$$
\begin{aligned}
M_{1} G_{5}\left(\mathscr{G}_{1}, x\right)= & 6 x^{24}+(4 t-4) x^{30}+6 x^{36}+8 x^{39}+(16 t-22) x^{42}+(8 t-12) x^{45} \\
& +\left(6 t^{2}-20 t+16\right) x^{48}+8 x^{51}+(8 t-14) x^{54}+(8 t-16) x^{57}+\left(6 t^{2}-24 t+24\right) x^{60}
\end{aligned}
$$

Also, from [4],

$$
\begin{aligned}
M_{2} G_{5}\left(\mathscr{G}_{1}, x\right)= & \sum_{r s \in E\left(\mathscr{G}_{1}\right)} x^{\left(S_{G}(r)+S_{G}(s)\right)} \\
& M_{2} G_{5}\left(\mathscr{G}_{1}, x\right)=x^{(6 \times 6)}\left|E_{1}\left(\mathscr{G}_{1}(t)\right)\right|+x^{(6 \times 11)}\left|E_{2}\left(\mathscr{G}_{1}(t)\right)\right|+x^{(6 \times 12)}\left|E_{3}\left(\mathscr{G}_{1}(t)\right)\right|+x^{(6 \times 14)}\left|E_{4}\left(\mathscr{G}_{1}(t)\right)\right| \\
& +x^{(7 \times 9)}\left|E_{5}\left(\mathscr{G}_{1}(t)\right)\right|+x^{(7 \times 12)}\left|E_{6}\left(\mathscr{G}_{1}(t)\right)\right|+x^{(8 \times 11)}\left|E_{7}\left(\mathscr{G}_{1}(t)\right)\right|+x^{(8 \times 13)}\left|E_{8}\left(\mathscr{G}_{1}(t)\right)\right| \\
& +x^{(9 \times 9)}\left|E_{9}\left(\mathscr{G}_{1}(t)\right)\right|+x^{(9 \times 14)}\left|E_{10}\left(\mathscr{G}_{1}(t)\right)\right|+x^{(11 \times 11)}\left|E_{11}\left(\mathscr{G}_{1}(t)\right)\right|+x^{(11 \times 12)}\left|E_{12}\left(\mathscr{G}_{1}(t)\right)\right| \\
& +x^{(11 \times 13)}\left|E_{13}\left(\mathscr{G}_{1}(t)\right)\right|+x^{(11 \times 14)}\left|E_{14}\left(\mathscr{G}_{1}(t)\right)\right|+x^{(11 \times 16)}\left|E_{15}\left(\mathscr{G}_{1}(t)\right)\right| \\
& +x^{(12 \times 14)}\left|E_{16}\left(\mathscr{G}_{1}(t)\right)\right|+x^{(13 \times 14)}\left|E_{17}\left(\mathscr{G}_{1}(t)\right)\right|+x^{(13 \times 16)}\left|E_{18}\left(\mathscr{G}_{1}(t)\right)\right| \\
& +x^{(14 \times 14)}\left|E_{19}\left(\mathscr{G}_{1}(t)\right)\right|+x^{(14 \times 16)}\left|E_{20}\left(\mathscr{G}_{1}(t)\right)\right| \\
= & x^{(6 \times 6)}(4 t)+x^{(6 \times 11)}(4 t)+x^{(6 \times 12)}(4)+x^{(6 \times 14)}(4 t-4)+x^{(7 \times 9)}(4 t-4)+x^{(7 \times 12)}(4 t-4) \\
& +x^{(8 \times 11)}(12 t-8)+x^{(8 \times 13)}(4 t-4)+x^{(9 \times 9)}(2 t-2)+x^{(9 \times 14)}(4 t-4) \\
& +x^{(11 \times 11)}\left(9 t^{2}-7 t+3\right)+x^{(11 \times 12)}(4)+x^{(11 \times 13)}(4 t-4)+x^{(11 \times 14)}\left(36 t^{2}-68 t+32\right) \\
& +x^{(11 \times 16)}(4 t-4)+x^{(12 \times 14)}(4 t-4)+x^{(13 \times 14)}(4 t-4)+x^{(13 \times 16)}(4 t-4) \\
& +x^{(14 \times 14)}(4 t-4)+x^{(14 \times 16)}\left(36 t^{2}-76 t+40\right) .
\end{aligned}
$$


By making some calculations, we obtain

$$
\begin{aligned}
M_{2} G_{5}\left(\mathscr{G}_{1}, x\right)= & 6 x^{144}+(4 t-4) x^{225}+6 x^{288}+8 x^{360}+8(2 t-3) x^{405}+2 x^{432}+4(2 t-3) x^{486} \\
& +2(t-2)(3 t-4) x^{540}+8 x^{648}+2(4 t-7) x^{729}+8(t-2) x^{810}+6(t-2)^{2} x^{900}
\end{aligned}
$$

Theorem 2.1.7. Let $\mathscr{G}_{1} \cong R H S L(t)$ be the rhombus-type silicate network; then, hyper-fifth M-Zagreb polynomials of first and second type are equal to

$$
\begin{aligned}
H M_{1} G_{5}\left(\mathscr{G}_{1}, x\right)= & 6 x^{576}+(4 t-4) x^{900}+6 x^{1296}+8 x^{1521}+(16 t-22) x^{1764}+(8 t-12) x^{2025} \\
& +\left(6 t^{2}-20 t+16\right) x^{2304}+8 x^{2601}+(8 t-14) x^{2916}+(8 t-16) x^{3249}+\left(6 t^{2}-24 t+24\right) x^{3600} \\
H M_{2} G_{5}\left(\mathscr{G}_{1}, x\right)= & 6 x^{20736}+(4 t-4) x^{50625}+6 x^{82944}+8 x^{129600}+(16 t-24) x^{164025} \\
& +2 x^{186624}+(8 t-12) x^{236196}+\left(6 t^{2}-20 t+16\right) x^{291600}+8 x^{419904} \\
& +(8 t-14) x^{531441}+(8 t-16) x^{656100}+\left(6 t^{2}-24 t+24\right) x^{810000}
\end{aligned}
$$

Proof. We obtain the outcome with the edge partition in

Table 1. It follows from [2] that

$$
\begin{aligned}
H M_{1} G_{5}\left(\mathscr{G}_{1}, x\right)= & \sum_{r s \in E\left(\mathscr{G}_{1}\right)} x^{\left(S_{G}(r)+S_{G}(s)\right)^{2}}, \\
H M_{1} G_{5}\left(\mathscr{G}_{1}, x\right)= & x^{(12+12)^{2}}\left|E_{1}\left(\mathscr{G}_{1}(t)\right)\right|+x^{(12+24)^{2}}\left|E_{2}\left(\mathscr{G}_{1}(t)\right)\right|+x^{(15+15)^{2}}\left|E_{3}\left(\mathscr{G}_{1}(t)\right)\right|+x^{(15+24)^{2}}\left|E_{4}\left(\mathscr{G}_{1}(t)\right)\right| \\
& +x^{(15+27)^{2}}\left|E_{5}\left(\mathscr{G}_{1}(t)\right)\right|+x^{(18+24)^{2}}\left|E_{6}\left(\mathscr{G}_{1}(t)\right)\right|+x^{(18+27)^{2}}\left|E_{7}\left(\mathscr{G}_{1}(t)\right)\right|+x^{(18+30)^{2}}\left|E_{8}\left(\mathscr{G}_{1}(t)\right)\right| \\
& +x^{(24+27)^{2}}\left|E_{9}\left(\mathscr{G}_{1}(t)\right)\right|+x^{(27+27)^{2}}\left|E_{10}\left(\mathscr{G}_{1}(t)\right)\right|+x^{(27+30)^{2}}\left|E_{11}\left(\mathscr{G}_{1}(t)\right)\right|+x^{(30+30)^{2}}\left|E_{12}\left(\mathscr{G}_{1}(t)\right)\right|, \\
= & x^{(12+12)^{2}}(6)+x^{(12+24)^{2}}(6)+x^{(15+15)^{2}}(4 t-4)+x^{(15+24)^{2}}(8) \\
& +x^{(15+27)^{2}}(16 t-24)+x^{(18+24)^{2}}(2)+x^{(18+27)^{2}}(8 t-12)+x^{(18+30)^{2}}\left(6 t^{2}-20 t+16\right) \\
& +x^{(24+27)^{2}}(8)+x^{(27+27)^{2}}(8 t-14)+x^{(27+30)^{2}}(8 t-16)+x^{(30+30)^{2}}\left(6 t^{2}-24 t+24\right) .
\end{aligned}
$$

By doing some calculations, we obtain

$$
\begin{aligned}
H M_{1} G_{5}\left(\mathscr{G}_{1}, x\right)= & 6 x^{576}+(4 t-4) x^{900}+6 x^{1296}+8 x^{1521}+(16 t-22) x^{1764}+(8 t-12) x^{2025} \\
& +\left(6 t^{2}-20 t+16\right) x^{2304}+8 x^{2601}+(8 t-14) x^{2916}+(8 t-16) x^{3249}+\left(6 t^{2}-24 t+24\right) x^{3600} .
\end{aligned}
$$

Also, from [10], 


$$
\begin{aligned}
H M_{2}^{a} G_{5}\left(\mathscr{G}_{1}, x\right)= & \sum_{r s \in E\left(\mathscr{G}_{1}\right)} x^{\left(S_{G}(r) \times S_{G}(s)\right)^{2}} \\
H M_{2}^{a} G_{5}\left(\mathscr{G}_{1}, x\right)= & x^{(12 \times 12)^{2}}\left|E_{1}\left(\mathscr{G}_{1}(t)\right)\right|+x^{(12 \times 24)^{2}}\left|E_{2}\left(\mathscr{G}_{1}(t)\right)\right|+x^{(15 \times 15)^{2}}\left|E_{3}\left(\mathscr{G}_{1}(t)\right)\right| \\
& +x^{(15 \times 24)^{2}}\left|E_{4}\left(\mathscr{G}_{1}(t)\right)\right|+x^{(15 \times 27)^{2}}\left|E_{5}\left(\mathscr{G}_{1}(t)\right)\right|+x^{(18 \times 24)^{2}}\left|E_{6}\left(\mathscr{G}_{1}(t)\right)\right| \\
& +x^{(18 \times 27)^{2}}\left|E_{7}\left(\mathscr{G}_{1}(t)\right)\right|+x^{(18 \times 30)^{2}}\left|E_{8}\left(\mathscr{G}_{1}(t)\right)\right|+x^{(24 \times 27)^{2}}\left|E_{9}\left(\mathscr{G}_{1}(t)\right)\right| \\
& +x^{(27 \times 27)^{2}}\left|E_{10}\left(\mathscr{G}_{1}(t)\right)\right|+x^{(27 \times 30)^{2}}\left|E_{11}\left(\mathscr{G}_{1}(t)\right)\right|+x^{(30 \times 30)^{2}}\left|E_{12}\left(\mathscr{G}_{1}(t)\right)\right| \\
= & x^{(12 \times 12)^{2}}(6)+x^{(12 \times 24)^{2}}(6)+x^{(15 \times 15)^{2}}(4 t-4)+x^{(15 \times 24)^{2}}(8)+x^{(15 \times 27)^{2}}(16 t-24) \\
& +x^{(18 \times 24)^{2}}(2)+x^{(18 \times 27)^{2}}(8 t-12)+x^{(18 \times 30)^{2}}\left(6 t^{2}-20 t+16\right)+x^{(24 \times 27)^{2}}(8) \\
& +x^{(27 \times 27)^{2}}(8 t-14)+x^{(27 \times 30)^{2}}(8 t-16)+x^{(30 \times 30)^{2}}\left(6 t^{2}-24 t+24\right) .
\end{aligned}
$$

By making some calculations, we obtain

$$
\begin{aligned}
H M_{2} G_{5}\left(\mathscr{G}_{1}, x\right)= & 6 x^{20736}+(4 t-4) x^{50625}+6 x^{82944}+8 x^{129600}+(16 t-24) x^{164025}+2 x^{186624} \\
& +(8 t-12) x^{236196}+\left(6 t^{2}-20 t+16\right) x^{291600}+8 x^{419904}+(8 t-14) x^{531441} \\
& +(8 t-16) x^{656100}+\left(6 t^{2}-24 t+24\right) x^{810000} .
\end{aligned}
$$

2.2. Results for the Rhombus Type of Oxide Networks. Now, we are calculating fifth M-Zagreb topological indices of the rhombus-type oxide network $\mathscr{G}_{2} \cong R \operatorname{HOX}(t)$, where $t \in \mathbb{N}$.

Theorem 2.2.1. Let $\mathscr{G}_{2} \cong R H O X(t)$ be the rhombus-type silicate network; then, the first and second fifth M-Zagreb indices are equal to

$$
\begin{aligned}
& M_{1} G_{5}\left(\mathscr{G}_{2}\right)=16\left(1-8 t+12 t^{2}\right) \\
& M_{2} G_{5}\left(\mathscr{G}_{2}\right)=16(2 t-1)(48 t-35) .
\end{aligned}
$$

Proof. The outcome can be obtained by using the edge partition in Table 2.

By using equation [5],

$$
\begin{aligned}
M_{1} G_{5}\left(\mathscr{G}_{2}\right)= & \sum_{r s \in E\left(\mathscr{G}_{2}\right)}\left(S_{G}(r)+S_{G}(s)\right), \\
M_{1} G_{5}\left(\mathscr{G}_{2}\right)= & (6+6)\left|E_{1}\left(\mathscr{G}_{2}(t)\right)\right|+(6+12)\left|E_{2}\left(\mathscr{G}_{2}(t)\right)\right|+(8+12)\left|E_{3}\left(\mathscr{G}_{2}(t)\right)\right|+(8+14)\left|E_{4}\left(\mathscr{G}_{2}(t)\right)\right| \\
& +(12+14)\left|E_{5}\left(\mathscr{G}_{2}(t)\right)\right|+(14+14)\left|E_{6}\left(\mathscr{G}_{2}(t)\right)\right|+(14+16)\left|E_{7}\left(\mathscr{G}_{2}(t)\right)\right|+(16+16)\left|E_{8}\left(\mathscr{G}_{2}(t)\right)\right|, \\
= & (6+6)(2)+(6+12)(4)+(8+12)(4)+(8+14)(8 t-12)+(12+14)(8)+(14+14) \\
& (8 t-14)+(14+16)(8 t-16)+(16+16)\left(6(t-2)^{2}\right) .
\end{aligned}
$$


TABLE 2: Edge partition of rhombus-type oxide network $(R H O X(t))$ based on sum of degrees of end vertices of each edge.

Where rs $\in E\left(\mathscr{G}_{2}\right)$

$(6,6)$

$(6,12)$

$(8,12)$

$(8,14)$

Where $r s \in E\left(\mathscr{G}_{2}\right)$

$(12,14)$

$(14,14)$

$(14,16)$

$(16,16)$

$$
\begin{gathered}
2 \\
4 \\
4 \\
4(2 t-3) \\
\\
8 \\
2(4 t-7) \\
8(t-2) \\
6(t-2)^{2}
\end{gathered}
$$

By doing some calculations, we obtain

$$
M_{1} G_{5}\left(\mathscr{G}_{2}\right)=16\left(1-8 t+12 t^{2}\right) .
$$

$$
\begin{aligned}
M_{2} G_{5}(\mathscr{G})= & \sum_{r s \in E(\mathscr{G})}\left(S_{G}(r)+S_{G}(s)\right), \\
M_{2} G_{5}\left(\mathscr{G}_{2}\right)= & (6+6)\left|E_{1}\left(\mathscr{G}_{2}(t)\right)\right|+(6+12)\left|E_{2}\left(\mathscr{G}_{2}(t)\right)\right|+(8+12)\left|E_{3}\left(\mathscr{G}_{2}(t)\right)\right|+(8+14)\left|E_{4}\left(\mathscr{G}_{2}(t)\right)\right| \\
& +(12+14)\left|E_{5}\left(\mathscr{G}_{2}(t)\right)\right|+(14+14)\left|E_{6}\left(\mathscr{G}_{2}(t)\right)\right|+(14+16)\left|E_{7}\left(\mathscr{G}_{2}(t)\right)\right|+(16+16)\left|E_{8}\left(\mathscr{G}_{2}(t)\right)\right|, \\
= & (6+6)(2)+(6+12)(4)+(8+12)(4)+(8+14)(8 t-12)+(12+14)(8)+(14+14) \\
& (8 t-14)+(14+16)(8 t-16)+(16+16)\left(6(t-2)^{2}\right),
\end{aligned}
$$

By doing some calculations, we obtain

$$
M_{2} G_{5}\left(\mathscr{G}_{2}\right)=16(2 t-1)(48 t-35) .
$$

Theorem 2.2.2. Consider the rhombus-type oxide network $\mathscr{G}_{2} \cong R H O X(t)$ for $t \in \mathbb{N}$. Then, the first and second general fifth $M$-Zagreb indices are equal to

$$
\begin{aligned}
M_{1}^{a} G_{5}\left(\mathscr{G}_{2}\right) & =\left[\begin{array}{r}
2^{a}\left(3 \times 2^{3+4 a}+2^{1+a} 3^{a}+2^{2+a} 5^{a}+4 \times 9^{a}-12 \times 11^{a}+8 \times 13^{a}-14^{1+a}-16 \times 15^{a}\right)+ \\
2^{a} t\left(-3 \times 2^{3+4 a}+2^{3+a} 7^{a}+8 \times 11^{a}+8 \times 15^{a}\right)+3 \times 2^{1+5 a} t^{2}
\end{array}\right], \\
M_{2}^{a} G_{5}\left(\mathscr{G}_{2}\right) & =\left[\begin{array}{r}
\left(3 \times 2^{3+8 a}+2^{2+5 a} 3^{a}-2^{4+5 a} 7^{a}-3 \times 4^{1+2 a} 7^{a}+2^{1+2 a} 9^{a}+2^{2+3 a} 9^{a}-14^{1+2 a}+8^{1+a} 21^{a}\right. \\
+t\left(-3 \times 2^{3+8 a}+2^{3+4 a} 7^{a}+2^{3+5 a} 7^{a}+2^{3+2 a} 49^{a}\right)+3 \times 2^{1+8 a} t^{2}
\end{array}\right] . \\
\text { Proof. Let } \mathscr{G}_{2} \text { be the rhombus-type oxide network. Table 2 } & M_{1}^{a} G_{5}(\mathscr{G})=\sum_{r s \in E(\mathscr{G})}\left(S_{G}(r)+S_{G}(s)\right)^{a} .
\end{aligned}
$$
shows such an edge partition of $\operatorname{RHOX}(t)$. Thus, from [9], it follows that
By using edge partitions in Table 2, we obtain

$$
\begin{aligned}
M_{1}^{a} G_{5}\left(\mathscr{G}_{2}\right)= & (6+6)^{a}\left|E_{1}\left(\mathscr{G}_{2}(t)\right)\right|+(6+12)^{a}\left|E_{2}\left(\mathscr{G}_{2}(t)\right)\right|+(8+12)^{a}\left|E_{3}\left(\mathscr{G}_{2}(t)\right)\right|+(8+14)^{a}\left|E_{4}\left(\mathscr{G}_{2}(t)\right)\right| \\
& +(12+14)^{a}\left|E_{5}\left(\mathscr{G}_{2}(t)\right)\right|+(14+14)^{a}\left|E_{6}\left(\mathscr{G}_{2}(t)\right)\right|+(14+16)^{a}\left|E_{7}\left(\mathscr{G}_{2}(t)\right)\right|+(16+16)^{a}\left|E_{8}\left(\mathscr{G}_{2}(t)\right)\right| \\
= & (6+6)^{a}(2)+(6+12)^{a}(4)+(8+12)^{a}(4)+(8+14)^{a}(8 t-12)+(12+14)^{a}(8)+(14+14)^{a} \\
& (8 t-14)+(14+16)^{a}(8 t-16)+(16+16)^{a}\left(6(t-2)^{2}\right) .
\end{aligned}
$$

By doing some calculations, we have 


$$
M_{1}^{a} G_{5}\left(\mathscr{G}_{2}\right)=\left[\begin{array}{c}
2^{a}\left(3 \times 2^{3+4 a}+2^{1+a} 3^{a}+2^{2+a} 5^{a}+4 \times 9^{a}-12 \times 11^{a}+8 \times 13^{a}-14^{1+a}-16 \times 15^{a}\right)+ \\
2^{a} t\left(-3 \times 2^{3+4 a}+2^{3+a} 7^{a}+8 \times 11^{a}+8 \times 15^{a}\right)+3 \times 2^{1+5 a} t^{2}
\end{array}\right] .
$$

From [12], we have

$$
M_{2}^{a} G_{5}(\mathscr{G})=\sum_{r s \in E(\mathscr{G})}\left(S_{G}(r)+S_{G}(s)\right)^{a} .
$$

By using edge partitions in Table 2, we obtain

$$
\begin{aligned}
M_{2}^{a} G_{5}\left(\mathscr{G}_{2}\right)= & (6+6)^{a}\left|E_{1}\left(\mathscr{G}_{2}(t)\right)\right|+(6+12)^{a}\left|E_{2}\left(\mathscr{G}_{2}(t)\right)\right|+(8+12)^{a}\left|E_{3}\left(\mathscr{G}_{2}(t)\right)\right|+(8+14)^{a}\left|E_{4}\left(\mathscr{G}_{2}(t)\right)\right| \\
& +(12+14)^{a}\left|E_{5}\left(\mathscr{G}_{2}(t)\right)\right|+(14+14)^{a}\left|E_{6}\left(\mathscr{G}_{2}(t)\right)\right|+(14+16)^{a}\left|E_{7}\left(\mathscr{G}_{2}(t)\right)\right|+(16+16)^{a}\left|E_{8}\left(\mathscr{G}_{2}(t)\right)\right| \\
= & (6+6)^{a}(2)+(6+12)^{a}(4)+(8+12)^{a}(4)+(8+14)^{a}(8 t-12)+(12+14)^{a}(8)+(14+14)^{a} \\
& (8 t-14)+(14+16)^{a}(8 t-16)+(16+16)^{a}\left(6(t-2)^{2}\right) .
\end{aligned}
$$

By doing some calculations, we have

$$
M_{2}^{a} G_{5}\left(\mathscr{G}_{2}\right)=\left[\begin{array}{l}
3 \times 2^{3+8 a}+2^{2+5 a} 3^{a}-2^{4+5 a} 7^{a}-3 \times 4^{1+2 a} 7^{a}+2^{1+2 a} 9^{a}+2^{2+3 a} 9^{a}-14^{1+2 a}+8^{1+a} 21^{a} \\
+t\left(-3 \times 2^{3+8 a}+2^{3+4 a} 7^{a}+2^{3+5 a} 7^{a}+2^{3+2 a} 49^{a}+3 \times 2^{1+8 a} t^{2}\right)
\end{array}\right]
$$

Theorem 2.2.3. Consider the rhombus-type oxide network $\mathscr{G}_{2} \cong R H O X(t)$ for $t \in \mathbb{N}$. Then, the first and second hyper fifth $M$-Zagreb indices are equal to

$$
\begin{aligned}
& H M_{1}^{a} G_{5}\left(\mathscr{G}_{2}\right)=64\left(31-113 t+96 t^{2}\right), \\
& H M_{2}^{a} G_{5}\left(\mathscr{G}_{2}\right)=192\left(1915-3978 t+2048 t^{2}\right) .
\end{aligned}
$$

Proof. Let $\mathscr{G}_{2}$ be the rhombus-type oxide network. Table 2 shows such an edge partition of $R H O X(t)$. Thus, from [13], it follows that

$$
H M_{1} G_{5}(\mathscr{G})=\sum_{r s \in E(\mathscr{G})}\left(S_{G}(r)+S_{G}(s)\right)^{2} .
$$

By using edge partitions in Table 2, we obtain

$$
\begin{aligned}
H M_{1} G_{5}\left(\mathscr{G}_{2}\right)= & (6+6)^{2}\left|E_{1}\left(\mathscr{G}_{2}(t)\right)\right|+(6+12)^{2}\left|E_{2}\left(\mathscr{G}_{2}(t)\right)\right|+(8+12)^{2}\left|E_{3}\left(\mathscr{G}_{2}(t)\right)\right|+(8+14)^{2}\left|E_{4}\left(\mathscr{G}_{2}(t)\right)\right| \\
& +(12+14)^{2}\left|E_{5}\left(\mathscr{G}_{2}(t)\right)\right|+(14+14)^{2}\left|E_{6}\left(\mathscr{G}_{2}(t)\right)\right|+(14+16)^{2}\left|E_{7}\left(\mathscr{G}_{2}(t)\right)\right|+(16+16)^{2}\left|E_{8}\left(\mathscr{G}_{2}(t)\right)\right|, \\
= & (6+6)^{2}(2)+(6+12)^{2}(4)+(8+12)^{2}(4)+(8+14)^{2}(8 t-12)+(12+14)^{2}(8)+(14+14)^{2} \\
& (8 t-14)+(14+16)^{2}(8 t-16)+(16+16)^{2}\left(6(t-2)^{2}\right) .
\end{aligned}
$$

By doing some calculations, we have

$$
H M_{1} G_{5}\left(\mathscr{G}_{2}\right)=64\left(31-113 t+96 t^{2}\right) \text {. }
$$

From [14], we have

$$
\begin{aligned}
H M_{2} G_{5}\left(\mathscr{G}_{2}\right)= & (6+6)^{2}\left|E_{1}\left(\mathscr{G}_{2}(t)\right)\right|+(6+12)^{2}\left|E_{2}\left(\mathscr{G}_{2}(t)\right)\right|+(8+12)^{2}\left|E_{3}\left(\mathscr{G}_{2}(t)\right)\right|+(8+14)^{2}\left|E_{4}\left(\mathscr{G}_{2}(t)\right)\right| \\
& +(12+14)^{2}\left|E_{5}\left(\mathscr{G}_{2}(t)\right)\right|+(14+14)^{2}\left|E_{6}\left(\mathscr{G}_{2}(t)\right)\right|+(14+16)^{2}\left|E_{7}\left(\mathscr{G}_{2}(t)\right)\right|+(16+16)^{2}\left|E_{8}\left(\mathscr{G}_{2}(t)\right)\right|, \\
= & (6+6)^{2}(2)+(6+12)^{2}(4)+(8+12)^{2}(4)+(8+14)^{2}(8 t-12)+(12+14)^{2}(8)+(14+14)^{2} \\
& (8 t-14)+(14+16)^{2}(8 t-16)+(16+16)^{2}\left(6(t-2)^{2}\right) .
\end{aligned}
$$


By doing some calculations, we have

$$
H M_{2} G_{5}\left(\mathscr{G}_{2}\right)=192\left(1915-3978 t+2048 t^{2}\right) \text {. }
$$

Theorem 2.2.4. Consider the rhombus-type silicate network $\mathscr{G}_{2} \cong R H O X(t)$ for $t \in \mathbb{N}$. Then, the third M-Zagreb index is equal to

$$
M_{3} G_{5}\left(\mathscr{G}_{2}\right)=(-48+64 t) \text {. }
$$

Proof. Let $\mathscr{G}_{2}$ be the rhombus-type oxide network. Table 2 shows such an edge partition of $R H O X(t)$. Thus, from [15], it follows that

$$
M_{3} G_{5}(\mathscr{G})=\sum_{r s \in E(\mathscr{G})}\left|S_{G}(r)-S_{G}(s)\right| .
$$

By using edge partitions in Table 2, we obtain

$$
\begin{aligned}
M_{3} G_{5}\left(\mathscr{G}_{2}\right)= & |6-6|\left|E_{1}\left(\mathscr{G}_{2}(t)\right)\right|+|6-12|\left|E_{2}\left(\mathscr{G}_{2}(t)\right)\right|+|8-12|\left|E_{3}\left(\mathscr{G}_{2}(t)\right)\right|+|8-14|\left|E_{4}\left(\mathscr{G}_{2}(t)\right)\right|+ \\
& |12-14|\left|E_{5}\left(\mathscr{G}_{2}(t)\right)\right|+|14-14|\left|E_{6}\left(\mathscr{G}_{2}(t)\right)\right|+|14-16|\left|E_{7}\left(\mathscr{G}_{2}(t)\right)\right|+|16-16 \|| E_{8}\left(\mathscr{G}_{2}(t)\right) \mid, \\
= & |6-6|(2)+|6-12|(4)+|8-12|(4)+|8-14|(8 t-12)+|12-14|(8)+|14-14| \\
& (8 t-14)+|14-16|(8 t-16)+|16-16|\left(6(t-2)^{2}\right) .
\end{aligned}
$$

By doing some calculations, we have

$$
M_{3} G_{5}\left(\mathscr{G}_{2}\right)=(-48+64 t) \text {. }
$$

Corresponding to the above indices, we are going to compute general fifth M-Zagreb polynomials for rhombus type of oxide network $R H O X(t)$.
Theorem 2.2.5. Let $\mathscr{G}_{2} \cong R H O X(t)$ be the rhombus-type oxide network; then, general fifth M-Zagreb polynomials of first and second type are equal to

$$
\begin{aligned}
M_{1}^{a} G_{5}\left(\mathscr{G}_{2}, x\right)= & 2 x^{12^{a}}+4 x^{18^{a}}+4 x^{20^{a}}+4(2 t-3) x^{22^{a}}+8 x^{26^{a}}+2(4 t-7) x^{28^{a}}+8(t-2) x^{30^{a}}+6(t-2)^{2} x^{32^{a}}, \\
M_{2}^{a} G_{5}\left(\mathscr{G}_{2}, x\right)= & 2 x^{36^{a}}+4 x^{72^{a}}+4 x^{96^{a}}+4(2 t-3) x^{112^{a}}+8 x^{168^{a}}+2(4 t-7) x^{196^{a}}+8(t-2) x^{224^{a}} \\
& +6(t-2)^{2} x^{256^{a}} .
\end{aligned}
$$

Proof. We obtain the outcome with the edge partition in

Table 1. It follows from [1] that

$$
\begin{aligned}
M_{1}^{a} G_{5}\left(\mathscr{G}_{2}, x\right)= & \sum_{r s \in E\left(\mathscr{G}_{2}\right)} x^{\left(S_{G}(r)+S_{G}(s)\right)^{a}} \\
M_{1}^{a} G_{5}\left(\mathscr{G}_{2}, x\right)= & x^{(6+6)^{a}}\left|E_{1}\left(\mathscr{G}_{2}(t)\right)\right|+x^{(6+12)^{a}}\left|E_{2}\left(\mathscr{G}_{2}(t)\right)\right|+x^{(8+12)^{a}}\left|E_{3}\left(\mathscr{G}_{2}(t)\right)\right|+x^{(8+14)^{a}}\left|E_{4}\left(\mathscr{G}_{2}(t)\right)\right| \\
& +x^{(12+14)^{a}}\left|E_{5}\left(\mathscr{G}_{2}(t)\right)\right|+x^{(14+14)^{a}}\left|E_{6}\left(\mathscr{G}_{2}(t)\right)\right|+x^{(14+16)^{a}}\left|E_{7}\left(\mathscr{G}_{2}(t)\right)\right|+x^{(16+16)^{a}}\left|E_{8}\left(\mathscr{G}_{2}(t)\right)\right| \\
= & x^{(6+6)^{a}}(2)+x^{(6+12)^{a}}(4)+x^{(8+12)^{a}}(4)+x^{(8+14)^{a}}(8 t-12)+x^{(12+14)^{a}}(8)+x^{(14+14)^{a}} \\
& (8 t-14)+x^{(14+16)^{a}}(8 t-16)+x^{(16+16)^{a}}\left(6(t-2)^{2}\right)
\end{aligned}
$$

By doing some calculations, we obtain

$$
M_{1}^{a} G_{5}\left(\mathscr{G}_{2}, x\right)=2 x^{12^{a}}+4 x^{18^{a}}+4 x^{20^{a}}+4(2 t-3) x^{22^{a}}+8 x^{26^{a}}+2(4 t-7) x^{28^{a}}+8(t-2) x^{30^{a}}+6(t-2)^{2} x^{32^{a}} .
$$

Also, from [3], 


$$
\begin{aligned}
M_{2}^{a} G_{5}\left(\mathscr{G}_{2}, x\right)= & \sum_{r s \in E\left(\mathscr{G}_{2}\right)} x^{\left(S_{G}(r)+S_{G}(s)\right)^{a}} \\
M_{2}^{a} G_{5}\left(\mathscr{G}_{2}, x\right)= & x^{(6+6)^{a}}\left|E_{1}\left(\mathscr{G}_{2}(t)\right)\right|+x^{(6+12)^{a}}\left|E_{2}\left(\mathscr{G}_{2}(t)\right)\right|+x^{(8+12)^{a}}\left|E_{3}\left(\mathscr{G}_{2}(t)\right)\right|+x^{(8+14)^{a}}\left|E_{4}\left(\mathscr{G}_{2}(t)\right)\right| \\
& +x^{(12+14)^{a}}\left|E_{5}\left(\mathscr{G}_{2}(t)\right)\right|+x^{(14+14)^{a}}\left|E_{6}\left(\mathscr{G}_{2}(t)\right)\right|+x^{(14+16)^{a}}\left|E_{7}\left(\mathscr{G}_{2}(t)\right)\right|+x^{(16+16)^{a}}\left|E_{8}\left(\mathscr{G}_{2}(t)\right)\right|, \\
= & x^{(6+6)^{a}}(2)+x^{(6+12)^{a}}(4)+x^{(8+12)^{a}}(4)+x^{(8+14)^{a}}(8 t-12)+x^{(12+14)^{a}}(8)+x^{(14+14)^{a}} \\
& (8 t-14)+x^{(14+16)^{a}}(8 t-16)+x^{(16+16)^{a}}\left(6(t-2)^{2}\right) .
\end{aligned}
$$

By making some calculations, we obtain

$$
\begin{aligned}
M_{2}^{a} G_{5}\left(\mathscr{G}_{2}, x\right)= & 2 x^{36^{a}}+4 x^{72^{a}}+4 x^{96^{a}}+4(2 t-3) x^{112^{a}}+8 x^{168^{a}}+2(4 t-7) x^{196^{a}}+8(t-2) x^{224^{a}} \\
& +6(t-2)^{2} x^{256^{a}} .
\end{aligned}
$$

Corresponding to the above indices, we are going to compute fifth $M$-Zagreb polynomials for rhombus-type oxide network $R H O X(t)$.
Theorem 2.2.6. Let $\mathscr{G}_{2} \cong R H O X(t)$ be the rhombus-type oxide network; then, fifth M-Zagreb polynomials of first and second type are equal to

$$
\begin{aligned}
& M_{1}^{a} G_{5}\left(\mathscr{G}_{2}, x\right)=2 x^{12}+4 x^{18}+4 x^{20}+4(2 t-3) x^{22}+8 x^{26}+2(4 t-7) x^{28}+8(t-2) x^{30}+6(t-2)^{2} x^{32}, \\
& M_{2} G_{5}\left(\mathscr{G}_{2}, x\right)=2 x^{36}+4 x^{72}+4 x^{96}+4(2 t-3) x^{112}+8 x^{168}+2(4 t-7) x^{196}+8(t-2) x^{224}+6 \\
& (t-2)^{2} x^{256} .
\end{aligned}
$$

Proof. We obtain the outcome with the edge partition in Table 2. It follows from [16] that

$$
\begin{aligned}
M_{1} G_{5}\left(\mathscr{G}_{2}, x\right)= & \sum_{r s \in E\left(\mathscr{G}_{2}\right)} x^{\left(S_{G}(r)+S_{G}(s)\right)}, \\
M_{1} G_{5}\left(\mathscr{G}_{2}, x\right)= & x^{(6+6)}\left|E_{1}\left(\mathscr{G}_{2}(t)\right)\right|+x^{(6+12)}\left|E_{2}\left(\mathscr{G}_{2}(t)\right)\right|+x^{(8+12)}\left|E_{3}\left(\mathscr{G}_{2}(t)\right)\right|+x^{(8+14)}\left|E_{4}\left(\mathscr{G}_{2}(t)\right)\right| \\
& +x^{(12+14)}\left|E_{5}\left(\mathscr{G}_{2}(t)\right)\right|+x^{(14+14)}\left|E_{6}\left(\mathscr{G}_{2}(t)\right)\right|+x^{(14+16)}\left|E_{7}\left(\mathscr{G}_{2}(t)\right)\right|+x^{(16+16)}\left|E_{8}\left(\mathscr{G}_{2}(t)\right)\right|, \\
= & x^{(6+6)}(2)+x^{(6+12)}(4)+x^{(8+12)}(4)+x^{(8+14)}(8 t-12)+x^{(12+14)}(8)+x^{(14+14)} \\
& (8 t-14)+x^{(14+16)}(8 t-16)+x^{(16+16)}\left(6(t-2)^{2}\right) .
\end{aligned}
$$

By doing some calculations, we obtain

$$
M_{1}^{a} G_{5}\left(\mathscr{G}_{2}, x\right)=2 x^{12}+4 x^{18}+4 x^{20}+4(2 t-3) x^{22}+8 x^{26}+2(4 t-7) x^{28}+8(t-2) x^{30}+6(t-2)^{2} x^{32} .
$$

Also, from [4], 


$$
\begin{aligned}
M_{2} G_{5}\left(\mathscr{G}_{2}, x\right)= & \sum_{r s \in E\left(\mathscr{G}_{2}\right)} x^{\left(S_{G}(r)+S_{G}(s)\right)} \\
M_{2} G_{5}\left(\mathscr{G}_{2}, x\right)= & x^{(6 \times 6)}\left|E_{1}\left(\mathscr{G}_{2}(t)\right)\right|+x^{(6 \times 12)}\left|E_{2}\left(\mathscr{G}_{2}(t)\right)\right|+x^{(8 \times 12)}\left|E_{3}\left(\mathscr{G}_{2}(t)\right)\right|+x^{(8 \times 14)}\left|E_{4}\left(\mathscr{G}_{2}(t)\right)\right|+ \\
& x^{(12 \times 14)}\left|E_{5}\left(\mathscr{G}_{2}(t)\right)\right|+x^{(14 \times 14)}\left|E_{6}\left(\mathscr{G}_{2}(t)\right)\right|+x^{(14 \times 16)}\left|E_{7}\left(\mathscr{G}_{2}(t)\right)\right|+x^{(16 \times 16)}\left|E_{8}\left(\mathscr{G}_{2}(t)\right)\right|, \\
= & x^{(6 \times 6)}(2)+x^{(6 \times 12)}(4)+x^{(8 \times 12)}(4)+x^{(8 \times 14)}(8 t-12)+x^{(12 \times 14)}(4)+x^{(14 \times 14)}(8 t-14) \\
& +x^{(14 \times 16)}(8 t-16)+x^{(16 \times 16)}\left(6(t-2)^{2}\right) .
\end{aligned}
$$

By making some calculations, we obtain

$$
\begin{aligned}
M_{2} G_{5}\left(\mathscr{G}_{2}, x\right)= & 2 x^{36}+4 x^{72}+4 x^{96}+4(2 t-3) x^{112}+8 x^{168}+2(4 t-7) x^{196}+8(t-2) x^{224} \\
& +6(t-2)^{2} x^{256}
\end{aligned}
$$

Theorem 2.2.7. Let $\mathscr{G}_{2} \cong R H O X(t)$ be the rhombus-type oxide network; then, hyper-fifth $M$-Zagreb polynomials of first and second type are equal to

$$
\begin{aligned}
H M_{1} G_{5}\left(\mathscr{G}_{2}, x\right)= & 2 x^{144}+4 x^{324}+4 x^{400}+4(2 t-3) x^{484}+8 x^{676} \\
& +2(4 t-7) x^{784}+8(t-2) x^{900}+6(t-2)^{2} x^{1024}, H M_{2} G_{5}\left(\mathscr{G}_{2}, x\right) \\
= & 2 x^{1296}+4 x^{5184}+4 x^{9216}+4(2 t-3) x^{12544}+8 x^{28224} \\
& +2(4 t-7) x^{38416}+8(t-2) x^{50176}+6(t-2)^{2} x^{65536} .
\end{aligned}
$$

Proof. We obtain the outcome with the edge partition in Table 2. It follows from [2] that

$$
\begin{aligned}
H M_{1} G_{5}\left(\mathscr{G}_{2}, x\right)= & \sum_{r s \in E\left(\mathscr{G}_{2}\right)} x^{\left(S_{G}(r)+S_{G}(s)\right)^{2}} \\
H M_{1} G_{5}\left(\mathscr{G}_{2}, x\right)= & x^{(6+6)^{2}}\left|E_{1}\left(\mathscr{G}_{2}(t)\right)\right|+x^{(6+12)^{2}}\left|E_{2}\left(\mathscr{G}_{2}(t)\right)\right|+x^{(8+12)^{2}}\left|E_{3}\left(\mathscr{G}_{2}(t)\right)\right|+x^{(8+14)^{2}}\left|E_{4}\left(\mathscr{G}_{2}(t)\right)\right| \\
& +x^{(12+14)^{2}}\left|E_{5}\left(\mathscr{G}_{2}(t)\right)\right|+x^{(14+14)^{2}}\left|E_{6}\left(\mathscr{G}_{2}(t)\right)\right|+x^{(14+16)^{2}}\left|E_{7}\left(\mathscr{G}_{2}(t)\right)\right|+x^{(16+16)^{2}}\left|E_{8}\left(\mathscr{G}_{2}(t)\right)\right| \\
= & x^{(6+6)^{2}}(2)+x^{(6+12)^{2}}(4)+x^{(8+12)^{2}}(4)+x^{(8+14)^{2}}(8 t-12)+x^{(12+14)^{2}}(8) \\
& +x^{(14+14)^{2}}(8 t-14)+x^{(14+16)^{2}}(8 t-16)+x^{(16+16)^{2}}\left(6(t-2)^{2}\right)
\end{aligned}
$$

By doing some calculations, we obtain

$$
\begin{aligned}
H M_{1} G_{5}\left(\mathscr{G}_{2}, x\right)= & 2 x^{144}+4 x^{324}+4 x^{400}+4(2 t-3) x^{484}+8 x^{676} \\
& +2(4 t-7) x^{784}+8(t-2) x^{900}+6(t-2)^{2} x^{1024} .
\end{aligned}
$$

Also, from [10], 


$$
\begin{aligned}
H M_{2}^{a} G_{5}\left(\mathscr{G}_{2}, x\right)= & \sum_{r s \in E\left(\mathscr{G}_{2}\right)} x^{\left(S_{G}(r)+S_{G}(s)\right)^{2}}, \\
H M_{2} G_{5}\left(\mathscr{G}_{2}, x\right)= & x^{(6 \times 6)^{2}}\left|E_{1}\left(\mathscr{G}_{2}(t)\right)\right|+x^{(6 \times 12)^{2}}\left|E_{2}\left(\mathscr{G}_{2}(t)\right)\right|+x^{(8 \times 12)^{2}}\left|E_{3}\left(\mathscr{G}_{2}(t)\right)\right|+x^{(8 \times 14)^{2}}\left|E_{4}\left(\mathscr{G}_{2}(t)\right)\right|+ \\
& x^{(12 \times 14)^{2}}\left|E_{5}\left(\mathscr{G}_{2}(t)\right)\right|+x^{(14 \times 14)^{2}}\left|E_{6}\left(\mathscr{G}_{2}(t)\right)\right|+x^{(14 \times 16)^{2}}\left|E_{7}\left(\mathscr{G}_{2}(t)\right)\right|+x^{(16 \times 16)^{2}}\left|E_{8}\left(\mathscr{G}_{2}(t)\right)\right|, \\
= & x^{(6 \times 6)^{2}}(2)+x^{(6 \times 12)^{2}}(4)+x^{(8 \times 12)^{2}}(4)+x^{(8 \times 14)^{2}}(8 t-12)+x^{(12 \times 14)^{2}}(4)+x^{(14 \times 14)^{2}}(8 t-14) \\
& +x^{(14 \times 16)^{2}}(8 t-16)+x^{(16 \times 16)^{2}}\left(6(t-2)^{2}\right) .
\end{aligned}
$$

By making some calculations, we obtain

$$
\begin{aligned}
H M_{2} G_{5}\left(\mathscr{G}_{2}, x\right)= & 2 x^{1296}+4 x^{5184}+4 x^{9216}+4(2 t-3) x^{12544}+8 x^{28224} \\
& +2(4 t-7) x^{38416}+8(t-2) x^{50176}+6(t-2)^{2} x^{65536}
\end{aligned}
$$

\section{Conclusion}

In this study, we computed sum of degree-based indices for $R H S L(t)$ and $R H O X(t)$ graphs of rhombus oxide and silicate structures. We also computed certain sum of degreebased polynomials such as fifth M-Zagreb, fifth hyper $\mathrm{M}$-Zagreb, and generalized fifth $\mathrm{M}$-Zagreb indices for $R H S L(t)$ and $R H O X(t)$ graphs of rhombus oxide and silicate structures. These facts may be useful for people working in computer science and chemistry fields who encounter chemical networks. These results can also play a vital role in the determination of the significance of silicate and oxide networks. Like certain other topological indices, determining the representations of derived graphs like these is an open question.

\section{Data Availability}

No data were used to support this study.

\section{Conflicts of Interest}

The authors declare no conflicts of interest.

\section{Acknowledgments}

This work was supported in part by the Natural Science Fund of Education Department of Anhui Province under Grant KJ2020A0478.

\section{References}

[1] A. Graovac, M. Ghorbani, and M. A. Hosseinzadeh, "Computing degree based topological properties of third type of hex-derived networks," Journal of Mathematical Nanoscience, vol. 1, pp. 33-42, 2011.

[2] V. R. Kulli, "General fifth M-zagreb indices and fifth M-zagreb polynomials of PAMAM dendrimers," International Journal of Fuzzy Mathematical Archive, vol. 22, pp. 99-103, 2017.
[3] M. Husin, R. Hasni, N. Arif, and M. Imran, "On topological indices of certain families of nanostar dendrimers," Molecules, vol. 21, no. 7, p. 821, 2016.

[4] V. R. Kulli, "Multiplicative hyper-Zagreb indices and coindices of graphs: computing these indices of some nanostructures," International Research Journal of Pure Algebra, vol. 6, no. 7, pp. 342-347, 2016.

[5] H. Ali, U. Babar, S. H. Arshad, and A. Sajjad, "On some neighbourhood degree-based indices of graphs derived from honeycomb structure," Konuralp Journal of Mathematics (KJM), vol. 9, no. 1, pp. 164-175, 2020.

[6] H. Ali, M. A. Binyamin, M. K. Shafiq, and W. Gao, "On the degree-based topological indices of some derived networks," Mathematics, vol. 7, no. 7, p. 612, 2019.

[7] W. Zhen, P. Ali, H. Ali, G. Dustigeer, and J. B. Liu, "On computation degree-based topological descriptors for planar octahedron networks," Journal of Mathematics, 2021.

[8] U. Babar, H. Ali, H. Ali, S. Hussain Arshad, and U. Sheikh, "Multiplicative topological properties of graphs derived from honeycomb structure," AIMS Mathematics, vol. 5, no. 2, pp. 1562-1587, 2020.

[9] M. V. Diudea, I. Gutman, and J. Lorentz, Molecular Topology, Nova Science Publishers, Huntington, NY, USA, 2001.

[10] A. Nayak and I. Stojmenovic, Hand Book of Applied Algorithms: Solving Scientific, Engineering, and Practical Problems, p. 560p, John Wiley \& Sons, Hoboken, NJ, USA, 2007.

[11] H. Wiener, "Structural determination of paraffin boiling points," Journal of the American Chemical Society, vol. 69, no. 1, pp. 17-20, 1947.

[12] M. Deza, P. W. Fowler, A. Rassat, and K. M. Rogers, "Fullerenes as tilings of surfaces," Journal of Chemical Information and Computer Sciences, vol. 40, no. 3, pp. 550-558, 2000.

[13] W. Gao, M. K. Siddiqui, M. Naeem, and M. Imran, "Computing multiple $\mathrm{ABC}$ index and multiple GA index of some grid graphs," Open Physics, vol. 16, no. 1, pp. 588-598, 2018.

[14] I. Gutman and S. J. Cyvin, Introduction to the Theory of Benzenoid Hydrocarbons, Springer, Berlin, Germany, 1989.

[15] I. Gutman, B. Rusi, and N. Trinajsti, "Graph theory and molecular orbitals. XII. Acyclic polyenes," The Journal of Chemical Physics, vol. 62, no. 9, pp. 3399-3405, 1975.

[16] M. Javaid, M. U. Rehman, and J. Cao, "Topological indices of rhombus type silicate and oxide networks," Canadian Journal of Chemistry, vol. 95, no. 2, pp. 134-143, 2017. 